\title{
Adenoid Variant of Basal Cell Carcinoma: A Case Report of a Rare Histopathological Subtype with Dermoscopic Aspects
}

\author{
Senhaji $\mathrm{G}^{1 *}$, Gallouj $\mathrm{S}^{1}$, Lamouaffaq $\mathrm{A}^{1}$, El Jouari $0^{1}$, Souaf $\mathrm{I}^{2}$ and \\ Mernissi FZ ${ }^{1}$ \\ ${ }^{1}$ Department of Dermatology, University Hospital Hassan II, Morocco \\ ${ }^{2} \mathrm{Al} \mathrm{Yosr} \mathrm{Center} \mathrm{of} \mathrm{Anatomopathology,} \mathrm{Morocco}$ \\ Case Report \\ Volume 3 Issue 1 \\ Received Date: January 20, 2018 \\ Published Date: February 06, 2018 \\ DOI: $10.23880 /$ cdoaj-16000140
}

*Corresponding author: Ghita senhaji, Department of Dermatology, University Hospital Hassan II, Fez, Morocco, Tel: 212600075857; E-mail: ghitasenhaji88@gmail.com

\begin{abstract}
Basal cell carcinoma (BCC) is the most common cutaneous tumor, accounting for approximately $70 \%$ of all malignant diseases of the skin. Ultraviolet radiation is considered the single most important risk factor for BCC. Thus, it arises commonly on sun-exposed sites such as the head and neck. Adenoid subtype is one such rare, indolent variant of conventional BCC, with an incidence of $1.3 \%$. It is often regarded as a low grade malignancy compared to other subtypes like nodular and morpheic form. We report here one such interesting very rare case of adenoid BCC of the malar region of the face with special regard to the dermoscopical and histological features.
\end{abstract}

Keywords: Basal cell carcinoma; Cutaneous tumor; Dermoscopy; Erythematous; Malar region

\section{Introduction}

Basal cell carcinoma (BCC), first described by Jacob in 1827 , is the most common cutaneous tumor, accounting for approximately $70 \%$ of all malignant diseases of the skin [1]. It is more prevalent after the fourth decade of life and its peak incidence is at the 6th decade with male preponderance [2]. Ultraviolet radiation is considered the single most important risk factor for BCC [3]. Thus, it arises commonly on sun-exposed sites such as the head and neck and occurs infrequently on non-sun-exposed sites [3]. BCCs can exhibit both a variety of growth patterns and a variety of types of differentiation [1]. Adenoid subtype is a rare histopathological variant which can morphologically present as pigmented and nonpigmented nodule or ulcer without predilection for any particular site [2]. Histological diagnosis and classifying BCCs into histological variants not only provide important information regarding low- and highrisk types of the malignancy but also has got a prognostic implication [4]. We report here one such interesting very rare case of adenoid BCC with it dermoscopical aspects.

\section{Case Report}

An 87-year-old male patient, with a history of chronic and intermittent solar exposure in childhood, presented with a 5-month history of a solitary painful, erythematous and well demarcated nodule, in the left malar region of the face. The size increased rapidly after chronic and 
repetitive manipulation of the lesion by hands, becoming oozing after 2 months. There was no previous personal or familial history of skin cancer and no other significant medical history was elicited.

Dermatological examination found a solitary, erythematous, budding and well-defined tumor of firm consistency, measuring about $2.5 \mathrm{~cm}$ at long axis, with smooth surface, sitting at the left malar region surrounded by multiple actinic keratoses on the face.

Dermoscopy showed a yellowish structure with erythematous areas, with polymorphic vascularization more marked at the periphery of the lesion and hemorrhagic suffusions. The local neurological examination was normal and no lymphadenopathy was appreciated.

A cutaneous biopsy confirmed the adenoid and cystic variant of basal cell carcinoma by showing a carcinomatous tumor proliferation connected to the epidermis, made of nodules and cords with a cyst-like enlarged structure, within a fibro inflammatory stroma. Peripheral pallisading with retraction spaces were seen all around. The cells were basaloid, with little atypical nuclei, fine chromatin and basophilic cytoplasm.
Based on clinical and histopathological findings, this case was diagnosed as an adenoid basal cell carcinoma. No extension assessment was necessary, and the patient had an excision of the entire lesion with safety margins. No recurrence was noted (Figures 1-3).

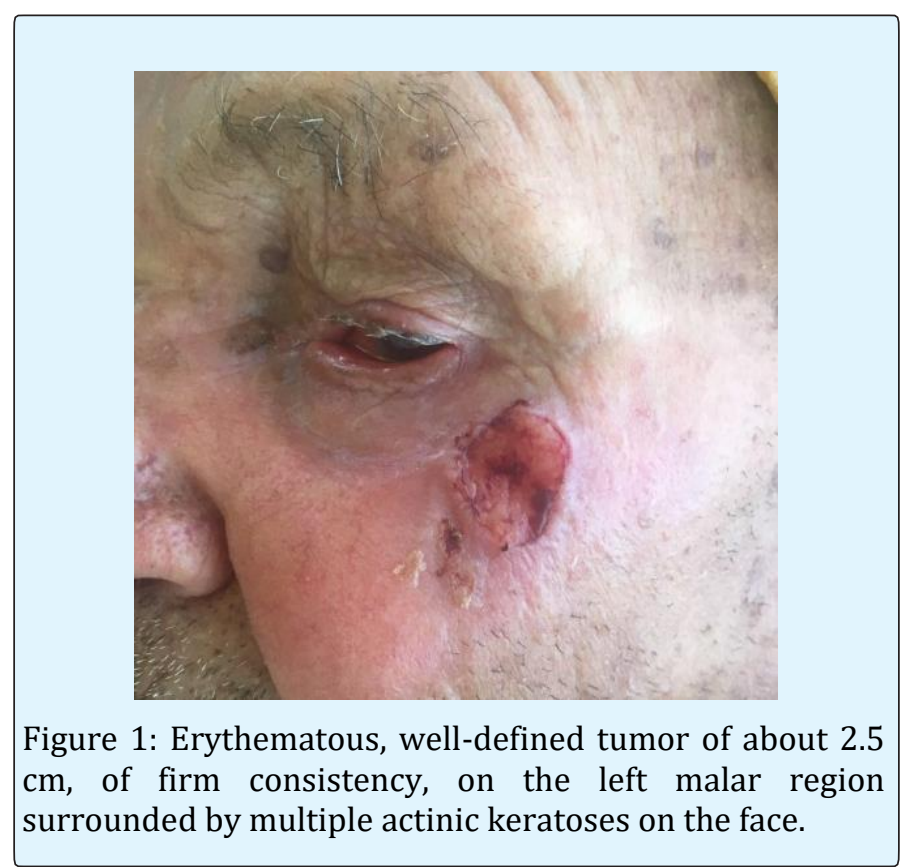

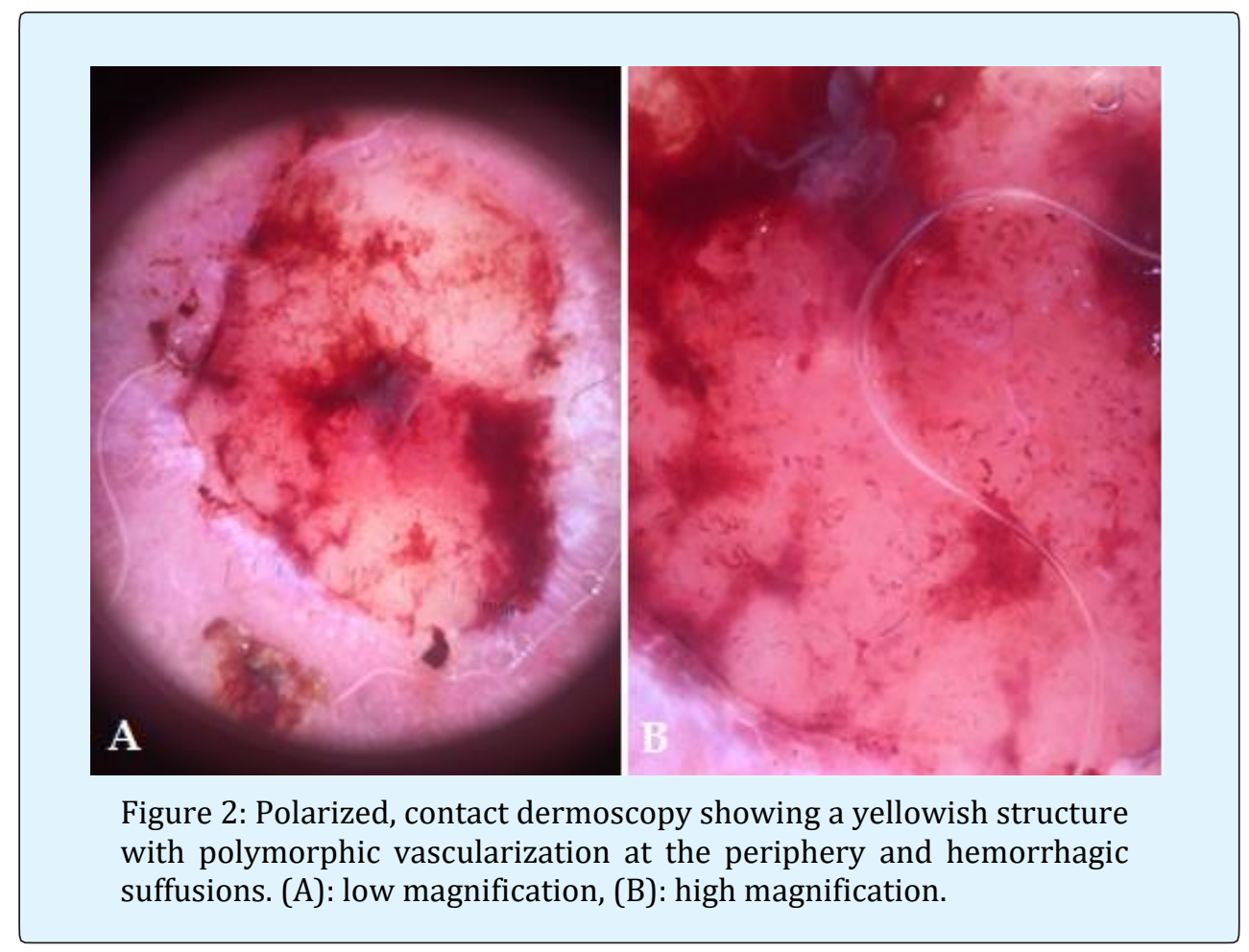

Senhaji G, et al. Adenoid Variant of Basal Cell Carcinoma: A Case Report of a Rare Histopathological Subtype with Dermoscopic Aspects. Clin Dermatol J 2018, 3(1): 000140. 


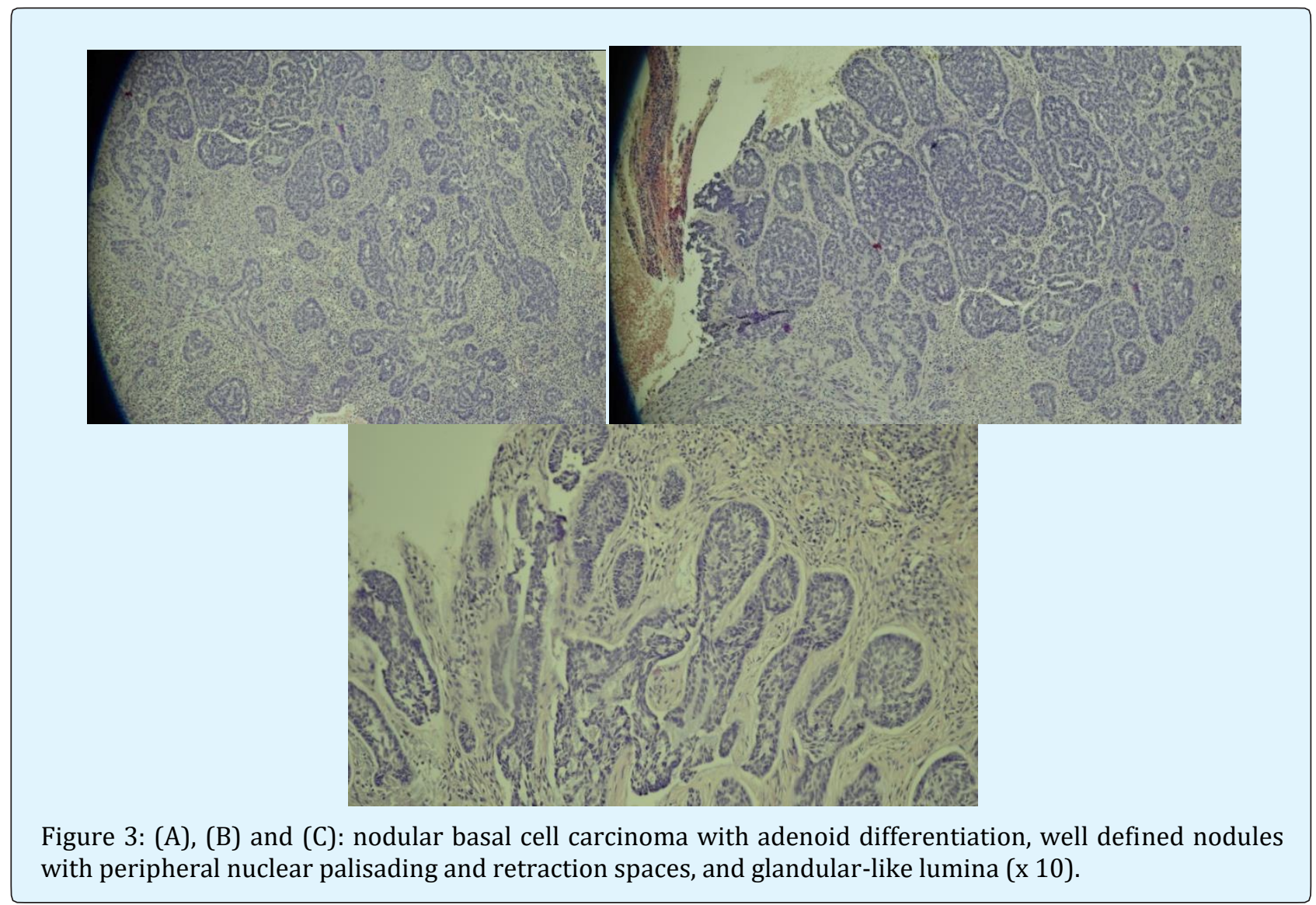

\section{Discussion}

BCC has been described as the most common malignant neoplasm of humans [1]. It is most located in head and neck region; the ages most often affected were decades VI and VII with a mean age of 61 years [5]. Recently, occurrence of BCC has increased [3]. The incidence of this type of cancer tends to increase with age, more than $90 \%$ of BCCs developing in people over 60 [3]. It most commonly occurs in sun-exposed sites such as the face and neck, where $80-90 \%$ of BCCs occur [2]. Although ultraviolet radiation is thought to be the primary risk factor in development of $\mathrm{BCC}$, the precise relationship is not as clear as with squamous cell carcinoma (SCC) [3]. As these tumours are rarely seen on forearm, hands and lower limbs, despite significant sun exposure [2]. In this case, a chronic and intermittent solar exposure in childhood was found as a major risk factor.

Rare morphological variants, such as cystic, adenoid, morphoeiform, infundibulocystic, pigmented and miscellaneous, together, account for less than $10 \%$ of all
BCCs [1]. Adenoid subtype is one such rare, indolent variant of conventional BCC [4]. It exact incidence is not known, but Patil, et al. reported the incidence of 1.3\% [4]. It is often regarded as a low grade malignancy compared to other subtypes like nodular and morpheic form [2], but has been reported to invade perivascular and perineural spaces in rare cases [6].

Adenoid BCC has been reported at various sites including axillae, back, leg, inner canthus of eye, chin and forehead and rarely even cervix and prostate [2]. Our patient had a BCC of the malar region of the face.

Histopathology of this rare variant shows arrangement of cells in the intertwining strands and radially around islands of connective tissue, resulting in a tumour with a lace like pattern [2]. It can also show a tumor composed of thin cords of interconnected basaloid cells achieving a reticular or glandular-like pattern [5]. Occasional cells surrounding these gland-like lumina may have a secretory appearance [6]. 


\section{Clinical Dermatology Open Access Journal}

Differential diagnosis of these tumors includes: cystic adenoid carcinoma (ACC) which often presents as a firm, slow growing, ill-defined nodule, the scalp being the most common site [1], with histology of carcinomatous proliferations with no connection to the epidermis and a constantly perineural invasion [5]. Another close mimic of adenoid BCC is primary cutaneous cribriform apocrine carcinoma, which is a rare low-grade cutaneous apocrine carcinoma [1], histologicaly showing a much more pleomorphic cellularity, with no connection to the epidermis, neither nuclear peripheral palisading pattern nor peritumoral stromal retractions [5].

The management of this adenoid type of basal cell carcinoma is similar to that of other types of basal cell carcinoma [2]. In the present case, surgical excisions with carcinological margins were performed.

Location, size, histological tumor type and treatment strategies are important factors in the recurrence of BCC [3]. Mainly, adenoid variant is regarded as a low-grade malignancy with low potential for recurrence and metastasis in majority of cases [4]. However, aggressive cases have been reported with invaded adjacent subcutaneous tissue, perivascular and perineural spaces [5]. In this case, no evidence of recurrence has been found during the follow-up visits until now.

\section{Conclusion}

To sum up, we present a new case of an adenoid and cystic histological variant of BCC, interesting by its extreme rarity and low aggressiveness both locally and generally. Through this case, we highlight the contribution of clinical and dermoscopical examination for the diagnostic orientation of this uncommon variety of BCC, with the histology being the cornerstone to confirm this diagnosis and to roul out differential diagnosis.

\section{References}

1. Jetley S, Jairajpuri ZS, Rana S, Talikoti MA (2013) Adenoid Basal Cell Carcinoma and its Mimics. Indian J Dermatol 58(3): 244.

2. Tambe SA, Ghate SS, Jerajani HR (2013) Adenoid Type of Basal Cell Carcinoma: Rare Histopathological Variant at an Unusual Location. Indian J Dermatol 58(2): 159.

3. Kim SH, Ko WT, Suh MK, Lee JI (2008) A Case of Axillary Adenoid Basal Cell Carcinoma. Ann Dermatol 20(1): 22-25.

4. Murkey N, Murkey P, Kalele KP, Patil KP (2017) Adenoid Variant of Basal Cell Carcinoma: A Case Report with a Glance at Biological Behavior of the Tumor. Indian J Dermatol 62(1): 103-105.

5. Mercu ŢR, Dast S, Ciurea ME, Mărgăritescu C, Popescu FD, et al. (2017) Histopathological aspects of some rare forms of facial basal cell carcinoma. Rom J Morphol Embryol 58(2): 425-432.

6. Golda N, Wheeland RG, Thomas TL, Trout C (2009) Adenoid Basal Cell Carcinoma Simulating Ameloblastoma. Dermatol Surg 35(9): 1410-1413. 